\title{
MAXIMUM LIKELIHOOD INFERENCE OF MOLECULAR PHYLOGENY
}

\author{
MASAMI HASEGAWA and HIROHISA KISHINO \\ The Institute of Statistical Mathematics \\ 4-6-7 Minami-Azabu, Minato-ku, Tokyo 106, JAPAN
}

\section{Introduction}

The evolutionary history of organisms is inscribed in the DNA of living organisms. Since the nucleotide substitution of DNA in evolution is best regarded as stochastic, statistical methods based on probabilistic models are required for inferring the history from the DNA sequences. Among these, a maximum likelihood method is on a firm theoretical basis of probability theory. It requires an explicit probabilistic model of nucleotide substitution to calculate the likelihood function and helps to make the assumptions clear.

In molecuar phylogenetics, we want to solve two problems; (1) inference of branching order, and (2) estimation of branching dates, and the two problems are interrelated. The estimation of branching dates has been based on an approximate constancy of the molecular evolutionary rate, that is called a molecular clock. However, it has recently been suggested that the rate sometimes differs among taxonomic units. A relative rate test is applicable to examine whether the rate differs among lineages (Hasegawa et al., 1987). The test, however, depends on the branching order. To avoide the circularity we should infer the branching order at first without assuming constancy of the rate. A maximum likelihood method developed by Felsenstein (1982) is suitable for this purpose (Hasegawa and Yano, 1984) and the method has been applied to a wide range of phylogenetic problems (Hasegawa et al., 1985a; Hasegawa and Kishino, 1989; Kishino and Hasegawa, 1989). Kishino et al. (1990) have developed a simple method for estimating a bootstrap probability of being the maximum likelihood tree among alternatives without performing maximum likelihood estimation for each resampled data.

After establishing the branching order, we shall estimate the branching dates by a method developed by Kishino and Hasegawa (1990) which is described briefly below. This is an extension of our earlier method based on the assumption of the rate constancy (Hasegawa et al., 1984, 1985b). The rate variation does not itself invalidate the molecular clock analysis, provided the variation can be incorporated into the model. The method has been applied to phylogenetic problems on man's place in the evolution of primates (長 谷川, 1989a, 1989b; Hasegawa 1990; Hasegawa and Kishino 1990; Hasegawa et al., 1989, 1990a) and on the root for polymorphisms of mitochondrial DNA in the present human population (Hasegawa and Horai, 1990).

Furthermore, we have developed a maximum likelihood method for inferring protein phylogeny from amino acid sequences (Kishino et al. 1990). The method has been applied to phylogenetic problems on the origin of chloroplasts (Kishino et al. 1990) and on the relationship among eukaryotes, archaebacteria and eubacteria (Hasegawa et al. 1990b). 


\section{A Statistical Model}

Let us consider $s$ homologous nucleotide sequences that consist of $n$ nuceotide sites. Our data set can be represented in table form as follows:

$$
\begin{array}{ccccccc}
X_{11}, & X_{12}, & X_{13} & \ldots, & X_{1 q} & \ldots, & X_{1 n} \\
X_{21}, & X_{22}, & X_{23} & \ldots, & X_{2 q} & \ldots, & X_{2 n} \\
\vdots & \vdots & \vdots & \ldots & \vdots & \ldots & \vdots \\
X_{p 1}, & X_{p 2}, & X_{p 3} & \ldots, & X_{p q} & \ldots, & X_{p n} \\
\vdots & \vdots & \vdots & \ldots & \vdots & \ldots & \vdots \\
X_{s 1}, & X_{s 2}, & X_{s 3} & \ldots, & X_{s q} & \ldots, & X_{s n}
\end{array}
$$

where $X_{p q}$ is either of $\mathrm{T}, \mathrm{C}, \mathrm{A}$, or $\mathrm{G}$, and shows the state of the $q$-th site of the $p$-th species. The table form of (1) can be rewritten as

$$
\mathrm{X}_{1}, \mathrm{X}_{2}, \mathrm{X}_{3}, \ldots, \mathrm{X}_{q}, \ldots, \mathrm{X}_{n}
$$

where $\mathrm{X}_{q}$ represents the $q$-th column of (1). There are $4^{s}$ possible outcomes for each site. Basic assumptions are that each site changes homogeneously and independently of others; that is, the process of nucleotide substitution follows an independently identical distribution (i.i.d.), and that the process is in equilibrium. Let $\mathrm{X}=\left(X_{1}, \ldots, X_{s}\right)^{T}$ (superscript $T$ denotes a transposed vector) be a representative variable of a site. From the data, we obtain information on the following probabilities:

$$
\operatorname{Pr}\left(X_{1}=i_{1}, \ldots, X_{s}=i_{s}\right)=P_{i_{1}, \ldots, i_{s}} \quad\left(i_{1}, \ldots, i_{s}=\mathrm{T}, \mathrm{C}, \mathrm{A}, \mathrm{G}\right) .
$$

We shall specify a model for the process of nucleotide substitution and represent these probabilities in terms of parameters such as the branching dates and substitution rates, and estimate the parameters by the maximum likelihood method.

We denote by $n_{i_{1}, \ldots, i_{s}}$, the number of sites that have a value of $\left(i_{1}, \ldots, i_{s}\right)$. This follows a multinomial ( $4^{s}$-nomial) distribution,

$$
\operatorname{Multi}\left(n ; P_{i_{1}, \ldots, i_{s}}, i_{1}, \ldots, i_{s}=\mathrm{T}, \mathrm{C}, \mathrm{A}, \mathrm{G}\right)
$$

and represents the most detailed information of the data under the assumption of an i.i.d. The average and the variance-covariance of these statistics are given by

$$
\begin{aligned}
E\left\{n_{i_{1}, \ldots, i_{s}}\right\} & =n P_{i_{1}, \ldots, i_{s}} \\
\operatorname{Cov}\left\{n_{i_{1}, \ldots, i_{s}}, n_{i_{1}^{\prime}, \ldots, i_{s}^{\prime}}\right\} & =n\left(\delta_{i_{1}, \ldots, i_{s} ; i_{1}^{\prime}, \ldots, i_{s}^{\prime}} P_{i_{1}, \ldots, i_{s}}-P_{i_{1}, \ldots, i_{s}} P_{i_{1}^{\prime}, \ldots, i_{s}^{\prime}}\right)
\end{aligned}
$$

where $\delta_{i_{1}, \cdots, i_{s} ; i_{1}^{\prime}, \cdots, i_{s}^{\prime}}$ equals 1 when $i_{1}=i_{1}^{\prime}, \ldots, i_{s}=i_{s}^{\prime}$ (variance), and 0 otherwise (covariance).

The number of states increases explosively as $s$ increases. Therefore, we shall reduce the data to differences, and compare the differences with a probability distribution to which they conform. 


\section{$2.1 \quad$ A Markov Model}

We assume that each site evolves according to a stationary Markov process in which a nucleotide $i$ (T, C, A, or $\mathrm{G}$ ) is replaced by another nucleotide $j$ in an infinitesimally short time interval, $d t$, with a probability of $P_{i j}(d t)$, as follows:

$$
P_{i j}(d t)=\left\{\begin{array}{lll}
\alpha \pi_{j} d t & \text { for transition } & (T \leftrightarrow C, A \leftrightarrow G) \\
\beta \pi_{j} d t & \text { for transversion } & (T, C \leftrightarrow A, G)
\end{array}\right.
$$

where $\pi_{j}$ is the composition of nucleotide $j$, and $\alpha$ and $\beta$ are parameters that determine transition rate and transversion rate, respectively (Hasegawa et al., 1984, 1985b).

Defining the substitution rate matrix $R$ by

$$
\begin{aligned}
& \begin{array}{llll}
T & C & A & G
\end{array} \\
& R=\begin{array}{c}
T \\
C \\
A \\
G
\end{array}\left(\begin{array}{cccc}
-\alpha \pi_{C}-\beta \pi_{R} & \alpha \pi_{C} & \beta \pi_{A} & \beta \pi_{G} \\
\alpha \pi_{T} & -\alpha \pi_{T}-\beta \pi_{R} & \beta \pi_{A} & \beta \pi_{G} \\
\beta \pi_{T} & \beta \pi_{C} & -\alpha \pi_{G}-\beta \pi_{Y} & \alpha \pi_{G} \\
\beta \pi_{T} & \beta \pi_{C} & \alpha \pi_{A} & -\alpha \pi_{A}-\beta \pi_{Y}
\end{array}\right),
\end{aligned}
$$

where $\pi_{Y}=\pi_{T}+\pi_{C}$ and $\pi_{R}=\pi_{A}+\pi_{G}$, the function $P(t)$ for an arbitrary time interval $t$ is given by

$$
P(t)=e^{t R}
$$

Denoting the eigenvalues and eigenvectors of $R$ by $\lambda_{i}$ and $u_{i}(i=1, \cdots, 4)$, and defining $U=\left(\mathrm{u}_{1}, \mathrm{u}_{2}, \mathrm{u}_{3}, \mathrm{u}_{4}\right)$, we can write the righthandside of the above equation as

$$
e^{t R}=U\left(\begin{array}{cccc}
e^{t \lambda_{1}} & & & \\
& e^{t \lambda_{2}} & & \\
& & e^{t \lambda_{3}} & \\
& & & e^{t \lambda_{4}}
\end{array}\right) U^{-1} .
$$

Now, the numbers of transition differences, $S_{i j}$, and transversion differences $V_{i j}$ between the $i$-th and $j$-th sequences are defined as follows:

$$
\begin{aligned}
& S_{i j}=\underbrace{\sum_{l_{1} l_{2}}}_{\text {transition }} n_{\ldots} \underbrace{}_{\hat{i}} l_{1} \ldots \underbrace{l_{2}}_{\hat{j}} \ldots, \\
& V_{i j}=\underbrace{\sum_{l_{1} l_{2}}}_{\text {transuersion }} n_{\ldots} \ldots \underbrace{l_{1}}_{\widehat{i}} \ldots \underbrace{l_{2}}_{\hat{j}} \ldots{ }^{\prime}
\end{aligned}
$$

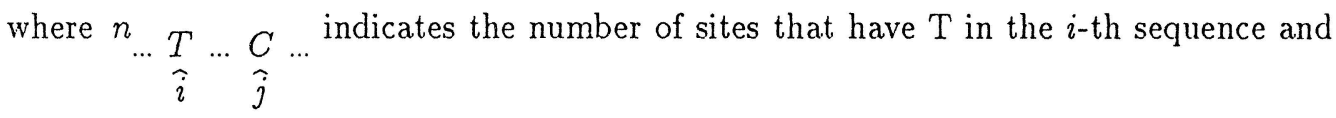

$\mathrm{C}$ in the $j$-th sequence irrespective of the other sequences.

Let us consider a pair of species $i$ and $j$ which separated $t$ million years (Myr) ago. When transition rate and transversion rate remain unchanged during evolution, from time reversibility and Chapman-Kolmogorov equation, the expected number of the transition and transversion differences between $i$ and $j$ are given by 


$$
\begin{gathered}
\overline{S_{i j}}=2 n\left\{\left(\pi_{T} \pi_{C}+\pi_{A} \pi_{G}\right)+\left(\pi_{T} \pi_{C} \pi_{R} / \pi_{Y}+\pi_{A} \pi_{G} \pi_{Y} / \pi_{R}\right) e^{-2 \beta t}\right. \\
\left.-\left(\pi_{T} \pi_{C} / \pi_{Y}\right) e^{2 t\left(\alpha \pi_{Y}+\beta \pi_{R}\right)}-\left(\pi_{A} \pi_{G} / \pi_{R}\right) e^{2 t\left(\alpha \pi_{R}+\beta \pi_{Y}\right)}\right\}, \\
\overline{V_{i j}}=2 n \pi_{Y} \pi_{R}\left\{1-e^{-2 \beta t}\right\} .
\end{gathered}
$$

When the evolutionary rate differs among different lineages, similar formulae can be derived (Kishino and Hasegawa, 1990).

\subsection{Likelihood Function}

The parameters are estimated by the maximum likelihood. From the central limit theorem, the random vector $\mathrm{D}=\left(V_{12}, \ldots, V_{1 s}, V_{23}, \ldots, V_{2 s}, \ldots, V_{(s-1) s}, S_{12}, \ldots\right.$, $\left.S_{1 s}, S_{23}, \ldots, S_{2 s}, \ldots, S_{(s-1) s}\right)^{T}$ follows asymptotically a multivariate normal distribution: $N(\overline{\mathrm{D}}, \Omega)$, where $\overline{\mathrm{D}}$ and $\Omega$ are the mean vector and the variance-covariance matrix of $\mathrm{D}$. Denoting the unknown parameters by $\theta=\left(t_{1}, t_{2}, t_{3}, \ldots, \alpha, \beta\right)^{T}$, the likelihood function is given by

$$
L(\theta \mid \mathrm{D})=\frac{1}{(2 \pi)^{s(s-1)} \sqrt{\operatorname{det} \Omega(\theta)}} \exp \left\{-\frac{1}{2}(\mathrm{D}-\overline{\mathrm{D}}(\theta))^{T} \Omega(\theta)^{-1}(\mathrm{D}-\overline{\mathrm{D}}(\theta))\right\}
$$

The maximum likelihood estimate of the parameter is denoted by $\widehat{\theta}$.

Defining sum of squares of residuals by

$$
R(\mathrm{D}, \theta)=(\mathrm{D}-\overline{\mathrm{D}}(\theta))^{T} \Omega^{-1}(\mathrm{D}-\overline{\mathrm{D}}(\theta))
$$

variance of the estimates $\operatorname{Var}(\hat{\theta})$ is given by

$$
\widehat{\operatorname{Var}}(\hat{\theta})=B^{-1}(\hat{\theta}) A(\hat{\theta}) \Omega(\hat{\theta}) A^{T}(\hat{\theta}) B^{-1}(\hat{\theta}) .
$$

where

and

$$
A(\theta)=\frac{\partial^{2}}{\partial \theta \partial \mathrm{D}^{T}} R(\theta \mid \mathrm{D})
$$

$$
B(\theta)=\frac{\partial^{2}}{\partial \theta \partial \theta^{T}} R(\theta \mid \mathrm{D})
$$

\subsection{Model Selection}

Different trce topologies constitute different models. Further, when we take into account of variable rate of substitution, several stages of heterogeneity among different branches can be considered. An extreme case is that every branch has its own parameters $\alpha_{k}$ and $\beta_{k}$, and the other extreme is rate constancy throughout the tree. If we introduce many parameters, we get a flexible model, but the precision of the estimates becomes worse. On the other hand, those models which are too simple give biased estimates. Minimizing both the bias and the variance is a trade-off requirement. It is important to adopt a 
criterion of model selection based on a firm theoretical background, the results of which are persuasive for almost everyone.

Based on the information theory, Akaike (1974) derived a criterion of model selection;

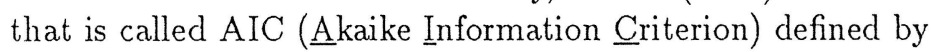

$$
\mathrm{AIC}=-2 \times(\text { estimated } \log \text { likelihood })+2 \times(\text { number of free parameters }),
$$

This is an asymptotically unbiased estimator of the expected Kullback-Leibler information quantity of the true distribution to the distribution estimated by the maximum likelihood method under the model. A model which minimizes the AIC is considered to be the most appropriate model. Minimum AIC estimate is a natural extension of the classical maximum likelihood estimate. It becomes possible to compare the adequacy of the approximation of different types of models even with different numbers of parameters.

\section{References}

Akaike, $\mathrm{H}$ (1974) A new look at the statistical model identification. IEEE Trans Autom Contr AC-19:716-723

Felsenstein J (1981) Evolutionary trees from DNA sequences: a maximum likelihood approach. J Mol Evol 17:368-376

Hasegawa M, Yano T (1984) Maximum likelihood method of phylogenetic inference from DNA sequence data. Bull Biomet Soc Jpn 5:1-7

Hasegawa M, Yano T, Kishino H (1984) A new molecular clock of mitochondrial DNA and the evolution of hominoids. Proc Jpn Acad 60B:95-98

Hasegawa M, Iida Y, Yano T, Takaiwa F, Iwabuchi M (1985a) Phylogenetic relationships among eukaryotic kingdoms inferred from ribosomal RNA sequences $J$ Mol Evol 22:32-38

Hasegawa M, Kishino H, Yano T (1985b) Dating of the human-ape splitting by a molecular clock of mitochondrial DNA. J Mol Evol 22:160-174

Hasegawa M, Kishino H, Yano T (1987) Man's place in Hominoidea as inferred from molecular clocks of DNA. J Mol Evol 26:132-147

長谷川政美 (1989a) D N A か みた人類の起原と進化一分子人類学序説 (增補版), 海鳴社 長谷川政美 (1989b) ヒトへの進化. 岩波講座一分子生物科学 3, 生物の歴史 (木村資生・大 沢省三編) pp.169-195

Hasegawa M, Kishino H (1989) Confidence limits on the maximum-likelihood estimate of the hominoid tree from mitochondrial-DNA sequences. Evolution 43:672-677

Hasegawa M, Kishino H, Yano T (1989) Estimation of branching dates among primates by molecular clocks of nuclear DNA which slowed down in Hominoidea. J Hum Evol 18:461-476

Hasegawa M (1990) Phylogeny and molecular evolution in primates. Jpn J Genet (in press)

Hasegawa M, Horai S (1990) Time of the deepest root for polymorphism in human mitochondrial DNA J Mol Evol (in press)

Hasegawa M and Kishino H (1990) DNA sequence analysis and evolution of Hominoidea. In: New Aspects of the Genetics of Molecular Evolution (eds: M. Kimura and N. Takahata), Japan Sci. Soc. Press, Tokyo (in press). 
Hasegawa M, Kishino H, Hayasaka K, Horai S (1990a) Mitochondrial DNA evolution in primates: transition rate has been extremely low in lemur. J Mol Evol 31 (in press)

Hasegawa M, Iwabe N, Mukohata Y and Miyata T (1990b) Close evolutionary relatedness of archaebacteria, Methanococcus and Halobacterium, to eukaryotes demonstrated by composite phylogenetic trees of elongation factors EF-Tu and EF-G: eocyte tree is unlikely. Jap J Genet 65, 109-114

Kishino H, Hasegawa M (1989) Evaluation of the maximum likelihood estimate of the evolutionary tree topologies from DNA sequence data, and the branching order in Hominoidea. J Mol Evol 29:170-179

Kishino H, Hasegawa M (1990) Converting distance to time: an application to human evolution. Methods Enz 183:550-570

Kishino H, Miyata T, Hasegawa M (1990) Maximum likelihood inference of protein phylogeny and the origin of chloroplasts. J Mol Evol 30 (in press)

[Received June, 1990] 